\title{
The Design and Implementation of Disaster Recovery in Dual-active Cloud Center
}

\author{
Xiao Chen ${ }^{1,}$, Longjun Zhang ${ }^{1, b}$ and Jiayong Chen $^{1}$ \\ ${ }^{1}$ Department of Information Engineering, Engineering University of CAPF, Xi'an 710086, China; \\ a1178200186@qq.com, 'longjun_zhang@sohu.com,
}

Keywords: Data center; Cloud disaster; dual-active; Virtualization; Storage policy.

\begin{abstract}
As Cloud computing has many advantages, it has been widely applied in various fields. As the increase of the number of users, the data volume will be more and more huge, The exponential growth of huge amounts of data causes that cloud disaster backup are increasingly important. This article is to focus on cloud computing environment of disaster recovery, combined with virtualization technology to design and implement scheme of "dual-active" solution and storage strategy based on" dual-active" data center. The network and business part is to realize the zero loss of user data and zero interruption of business.
\end{abstract}

\section{Introduction}

In recent years, with the rapid development of virtualization and network technique, cloud computing is quietly emerging within the scope of world and causes the third information technological revolution. Cloud computing provides a brand-new informational service pattern with tremendous market space. With the gradual mature and development of cloud computing, its economic benefit is also increasingly obvious. Meanwhile, appearance of cloud storage technology also offers a third-party IT resource and service with required demand and elastic framework to users and can effectively solve problems of continuous expansion of IT resource demand brought by rapid development of information technology.

However, the value of cloud computing and cloud storage is so tremendous, but lots of users still wander between cloud computing and traditional software architecture. The primary cause lies in inappropriate solution for the problem of user data integrity and availability in cloud computing field. It can say that the problem of data integrity and availability is the biggest obstacle to transfer data and application to cloud computing framework by users, while user data integrity and availability depend on disaster recovery system extremely. Therefore, in existing environment, it also proposes a new height to data security of IT system. Data disaster recovery in traditional way can't satisfy disaster recovery requirements in the new environment any more. Data valid storage of cloud disaster recovery has become a great bottleneck of developing disaster recovery. It is an urge demand to construct a kind of new-type "active-active" data center in cloud disaster recovery environment. This paper mainly introduces to construct "active-active data center based on traditional network framework", designs and realizes the "active-active” scheme of network and business, and achieves no loss of user data and zero interruption of business by constructing virtual experimental environment.

\section{Cloud Computing and Cloud Disaster Recovery}

Cloud Computing System.Liu Peng, an expert of Chinese grid computing and cloud computing, defines the cloud computing as follows: "Cloud computing distributes calculation tasks into lots of resource pools constituted by lots of computers and makes various application systems obtain computing power, storage space and all kinds of software services in line with demands” [1]. Simply speaking, cloud computing is a kind of pattern that makes use of large-scale data center and super computer cluster to establish enormous arithmetic system, makes users can obtain required various 
software and services through the internet and has no need to purchase independent operation system and various software for every microcomputer.

Cloud calculation system structure is consisted of 5 main parts, including application layer, platform layer, resource layer, visit to user layer and management layer, respectively. The essence of cloud computing is to provide service through network, so its system structure is service-centered. Application layer, platform layer and resource layer is the service with different levels offered by cloud computing, as shown in Fig.1:

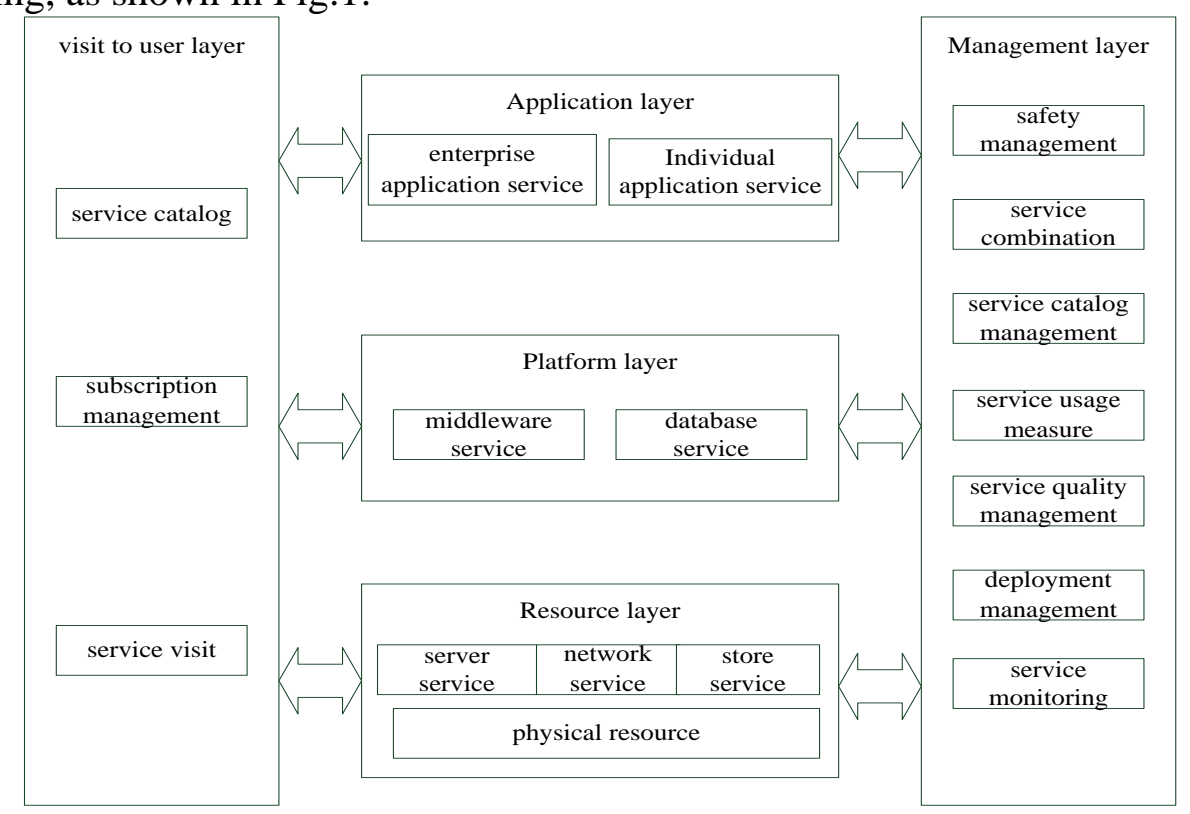

Fig.1 Cloud Computing System

Resource pool layer refer to the cloud computing service in infrastructures. This service can provide virtualized resources, so as to hide complexity of physical resource. Platform layer provides packaging of resource layer service for users and makes users use more advanced service to construct their own application. Application layer offers software service for users. Visit to user layer is convenient for users to utilize various supporting services required by cloud computing service and supply corresponding interface by aiming at cloud computing at each level. Management layer offers management functions of all levels in cloud computing service.

Cloud Disaster Recovery. Cloud disaster recovery is a newly-developing concept in disaster recovery field. Its advent provides an effective solution of disaster recovery for enterprises. Cloud disaster recovery refers to a pattern that it regards disaster recovery as a kind of service, the third-party manufacturer who establishes cloud provides infrastructures and clients pay for providers to use disaster recovery service [2, 3]. By adopting this pattern, clients can make use of excellent technological resources, abundant disaster recovery experience and mature operation and maintenance management process of service providers to realize clients' disaster recovery targets rapidly, reduce clients' operation and maintenance cost and working strength, and lower total possession cost of disaster recovery system.

Traditional cloud disaster service adopts current advanced, safe and reliable data backup and data reproduction technology to establish manageable and operable disaster recovery service and provide disaster recovery service with different ranks in the same city or different places for users, so as to ensure that it can recover client business data and key application system rapidly and accurately after disaster, as well as continuous operation of client business. At present, cloud disaster recovery service mainly includes data disaster recovery and application disaster recovery.

Cloud disaster recovery has bright advantages of high degree of specialization, lower cost investment, resource sharing and high service quality. Such an advantage endows powerful life force for "main current" of socialized service system, like cloud disaster recovery, etc. Based on America, where has mature disaster recovery industry, cloud disaster recovery has already occupied 56\% of 
disaster recovery industry. Thus it can be seen that cloud disaster recovery is becoming a mainstream tendency [4].

\section{"Dual-active" data center}

Data center is the place with the most intensive information system resources and the most frequent data exchange, which can provide cyber source, service hosting and broadband access and many other network services to users [5]. With the deepening of the application of cloud computing, data center operation environment is changed from traditional client / server to network-connected large-scale server cluster.

"Dual-active" data center came into being for the purpose of disaster recovery of "the cloud" [6]. Broadly it refers to that the two data centers are in the running state, can undertake business, improve the utilization rate of data center's overall service capacity and system resources, and can have the mutual backup of the two data centers. When a fault happens to the data center which is providing the business service, business can automatically switches to the other data center, to achieve zero loss of the data and zero interrupt of the service.

"Dual-active" data center construction is a complicated system engineering, including several aspects of data, system, business, network, server and other infrastructure resources, and many of the needs of users are mixed together. The requirement of building extraordinarily complex, "dual-active " data center is mainly reflected in three aspects of the link of wide area network, the server / storage and the basic infrastructure of the machinery room. According to different requirements, data center deployment model includes network dual activity, service dual activity and resource dual activity and so on. There is no necessary link among the three, so they can undertake independent construction, and can also be combined to undertake the construction to meet the requirements for performance, investment protection and service deployment flexibility of different users. This paper will study and design the plan of the "dual activity" of network and business.

\section{"Dual-active" service design and realization on the basis of cloud computing}

"Dual-activity" of network and business. Under the premise that business supports "dual activity", the realization of the network of " dual activity " needs that the address sections that "dual activity" business allocates in different data centers are different [7]. The program design uses and DNS and the network technology with balanced global load, as shown in Fig.2:

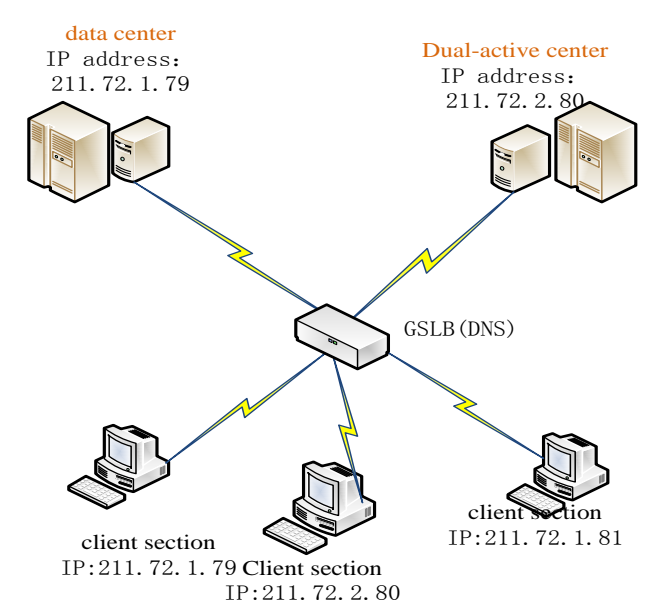

Fig.2 The "dual active” deployment scheme of network and business 
Data center and dual active center can be in independent response to the business, but they are distributed in different segments. Because the server can configure different gateway addresses, the model requires the same kind of business of the service system support the IP addresses and gateway addresses of different network segments, and through the DNS and global load balancing technology to ensure that the clients can automatically visit the site with best performance and the consistency of business.

The front end of data center adopts GSLB (global server load balance) based on intelligent DNS analysis, whose main task is to finish the load balance of DNS analysis request, the server status monitor and the optimization of client access path to guarantee distribute the request of the customers to available node [8]. Domain name resolution request is in the charge of GSLB, and through a set of predefined strategy, provided to the customers with the nearest node address to ensure a fast service. GSLB using the way of DNS can timely find business interruption and automatically switch to the site which can be visited. In order to ensure the sustainable business supply ability of the "dual-active" data center, server load balancing and HA technology should be used in the back end to cooperate with the dual activity of the front end network to realize service resource allocation and high availability.

The storage service design Based on the "dual-active" center. On the basis of cloud computing network and service "dual activity", design and implement the storage service solution based on "dual activity", as shown in Fig.3:

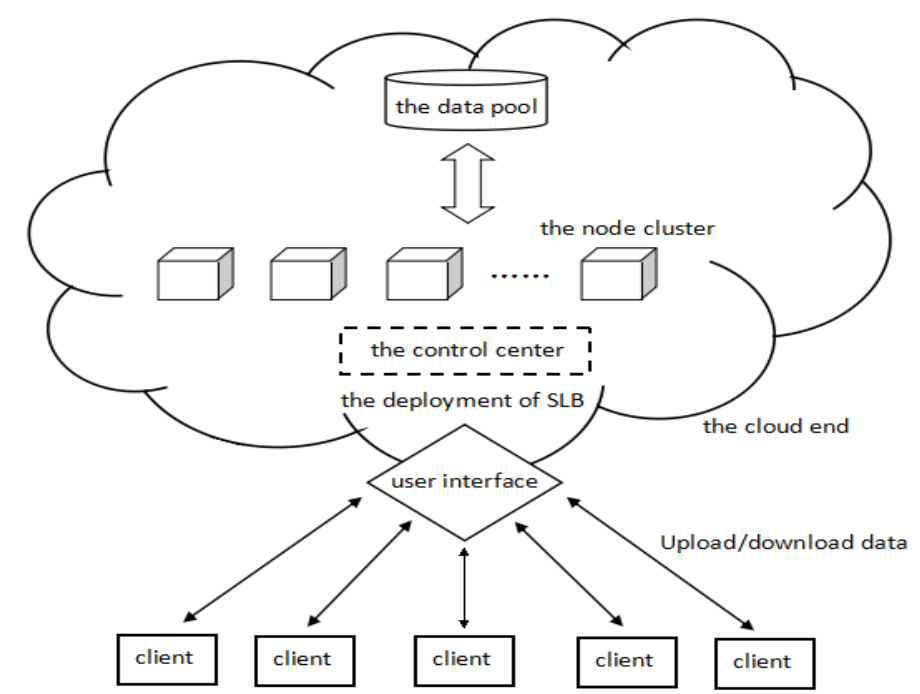

Fig.3 The storage service design of the data center

The plan adopts server virtualization and storage virtualization, virtual resource pool and dynamic application and data movement. The storage virtualization is achieved through VPLEX and server virtualization is achieved through the VMware. Through the interface of the client, the client realizes the upload and download of the data. The data pool is only a place for data storage, which stores all data of the clients. The data pool itself does not need to provide calculable capability.

In order to meet the service needs of high performance and high reliability, the operational parts of the cloud are concentrated in the node. The node group is a cluster of virtualization to carry out the virtualization through virtual technology, and every virtual server provides the same or similar network storage service, and receives the management of the control center which is located in the front of the node cluster. The control center deploys SLB [9] and is responsible for the distribution of the customers' request in a server cluster according to the equilibrium strategy, provides the customers with service and keeps the maintenance of the server availability. In cooperation with GSLB in the front end of data center, SLB can realize the load balancing from the front end to the data center internal full path and better realize the health detection of the servers In the process of calculation, the nodes are dynamically adjusted to elastic telescopic cloud, responsible for handling the client data, and finally be saved to a data pool. Between the main data center and the "dual-active" 
data center, two layers of Internet are needed to meet the two-layer communication requirements of the members of the cluster, and at the same time, SAN interconnection is also needed to realize the data synchronization.

\section{Experimental simulation}

Under laboratory environment, 3 servers, 16 computers, 4 routers and other hardware equipments are used to conduct mock implementation for data center system solutions of cloud computing architecture. Among which, the data is distributed on the server with two different network segments, and with sub-module system in its architecture to simulate cloud data center. And there has another management server, which is control center and 16 computers are client. Simulation experiment result is shown in Fig.4:

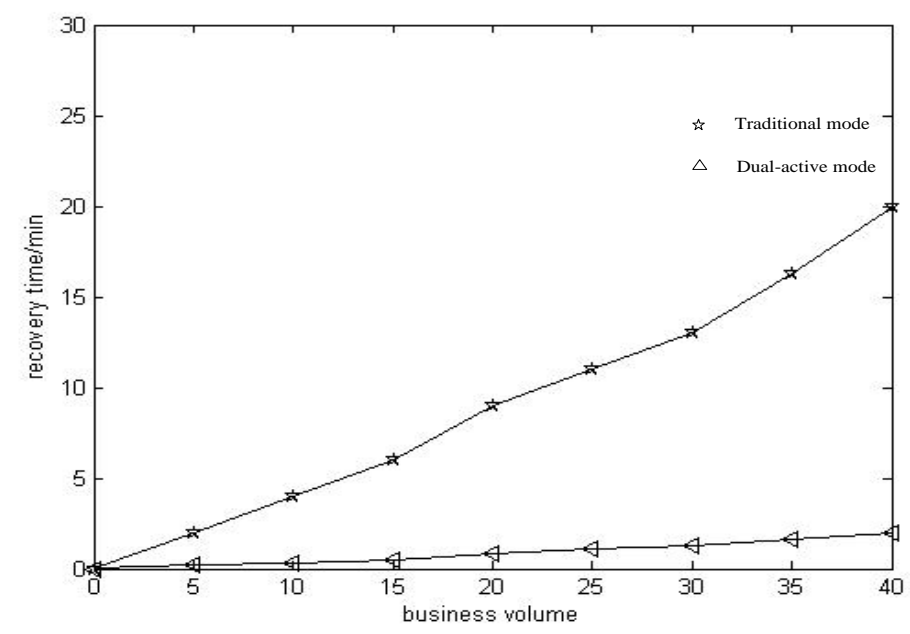

Fig. 4 The simulation results

As can be seen from the above figure, if traditional disaster recovery program is used, when a failure happens on main data center, business recovery time significantly gets longer as the increase of business volume; as regards disaster recovery system which is deployed with "dual-active" program, with the increase of business volume, business interruption time is very short after main data center stops working. The experiment result shows that "dual-active" data center can better achieve disaster recovery result.

\section{Summary}

As a new disaster recovery form, cloud disaster preparedness has gained wide attention, however, rapid growth of the data has made traditional cloud disaster preparedness fail to adapt to development needs of information age. Under such circumstances, "dual-active” data center rises in response to the "cloud", which helps to solve shortcomings of traditional disaster recovery way.

Aiming at cloud computing environment, this paper designs "dual-active” of network and business, as well as storage program based on "dual-active” center. However, "dual-active” data center of a real sense actually also contains dual-active of data, owing to current technical restriction, data consistency guarantees and other reasons, data center in "dual-active" program is still in an "Active" state, other data centers choose synchronization replication or asynchronous replication on the basis of distance to realize data backup function. So "dual-active" data center program discussed in this paper is only involved in infrastructure and the implemented "dual-active". At the same time, direction of future data center construction and technology selection should comply with virtualization, cloud computing and other trends, in addition, should fully consider standard, openness, flexibility, maintainability and other factors at the time of choosing technology.

\section{References}


[1] Peng Liu, Cloud Computing, M. Peking: Electronic Industry Press, 2011.

[2] A. Bajpai, P. Rana, and S. Maitrey, Remote mirroring:A disaster recovery technique in cloud computing, International Journal of Advance Research in Science and Engineering, vol. 2, no. 8, 2013. [3] Yu Gu, Dongsheng Wang, and Chuanyi Liu, DR-Cloud: Multi-Cloud Based Disaster Recovery Service, Tsinghua Science and Technology ISSN 1007-0214 pp13-23 Volume 19, Number 1, February 2014.

[4] Ferdaus, M. H., \& Murshed, M. Energy-Aware Virtual Machine Consolidation in IaaS Cloud Computing Cloud Computing, 2014, pp. 179-208.

[5] Kamal, J. M. M., \& Murshed, M. Distributed Database Management Systems: Architectural Design Choices for the Cloud Computing, 2014, pp. 23-50.

[6] Kiran, M. A Methodology for Cloud Security Risks Management Cloud Computing, 2014, pp. 75-104.

[7] Kronabeter, A., \& Fenz, S. Cloud Security and Privacy in the Light of the 2012 EU Data Protection Regulation Cloud Computing, 2013, pp. 114-123.

[8] Lombardi, F., \& Di Pietro, R. Virtualization and Cloud Security: Benefits, Caveats, and Future Developments Cloud Computing, 2014, pp. 237-255.

[9] Peng, G. C. A., Dutta, A., \& Choudhary, A. Exploring critical risks associated with enterprise cloud computing Cloud Computing, 2014, pp. 132-141.

[10] Wei Den, Fangming Liu, Hai Jin, et al. Leveraging Renewable Energy in Cloud Computing Datacenters :State of the Art and Future Research J. Chinese Journal of computer, 03(2013),582-598.

[11] Information on http://aws.amazon.com/simpledb/. 